\title{
The expression pattern of galectins during human epidermal morphogenesis
}

\author{
Efstathia Pasmatzi ${ }^{1}$, Christina Papadionysiou ${ }^{1}$, Alexandra Monastirli ${ }^{1}$, Stavros Kakkos ${ }^{2}$, George Badavanis ${ }^{1}$, George \\ Adonakis³, George Stamatiou4 ${ }^{4}$, Dionysios Tsambaos ${ }^{1}$
}

${ }^{1}$ Department of Dermatology, School of Medicine, University of Patras, Patras, Greece. ${ }^{2}$ Department of Vascular Surgery, School of Medicine, University of Patras, Patras, Greece. ${ }^{3}$ Department of Obstetrics and Gynecology, School of Medicine, University of Patras, Patras, Greece. ${ }^{4}$ Department of Gynecology and Obstetrics, Iasso General Hospital, Athens, Greece.

\begin{abstract}
Introduction: Galectins constitute a phylogenetically conserved family of proteins with high binding affinity for glycoconjugates bearing $\beta$-galactoside residues. Surprisingly, knowledge of the expression pattern of galectins during human epidermal morphogenesis is very limited.

Methods: Fifty-eight biopsy skin specimens obtained from human embryos and 10 biopsy specimens obtained from healthy adult volunteers were processed for immunohistochemistry using a panel of antibodies against galectins 1, 3, 7, and 9.

Results: Fetal human epidermis was devoid of any galectin 1 immunoreactivity, whereas clear-cut changes were found in the galectin 3 immunoreactivity of fetal epidermis with advancing gestational age. The expression pattern of galectins 7 and 9 remained constant at all stages of gestation.

Conclusions: The changes in galectin 3 immunoreactivity of human fetal epidermis with advancing gestational age, which are reported here for the first time, suggest that this galectin and its ligands may be implicated in the molecular events underlying human epidermal morphogenesis. It remains to be elucidated in future investigations whether the expression pattern of galectins, particularly that of galectin 3 , in the developing human epidermis reveals alterations in fetuses with inherited cutaneous disorders that may be important for prenatal diagnosis of these disorders.
\end{abstract}

Keywords: galectin 1, galectin 3, galectin 7, galectin 9, fetal human epidermis

Received: 15 March 2020 | Returned for modification: 11 May 2020 | Accepted: 9 June 2020

\section{Introduction}

Galectins constitute a phylogenetically conserved family of nonglycosylated soluble proteins that are expressed in a wide range of species and show a high binding affinity for glycoconjugates bearing $\beta$-galactoside residues. All galectins contain one or two conserved carbohydrate recognition domains (CRDs) of about 130 amino acids that are responsible for $\beta$-galactoside binding (1). Based on their structural characteristics and particularly on the CRD organization, galectins can be classified into three types: 1) the "proto-type," containing one CRD, 2) the chimera type, containing a single CRD motif combined with a proline-rich N-terminal domain, and 3) the tandem-repeat-type, consisting of two CRDs separated by a short linker sequence (2).

Although CRDs of all galectins reveal an affinity for lactose and/or the saccharide ligand $\mathrm{N}$-acetyl-lactosamine, individual galectins are also capable of recognizing modifications of the latter and thus exhibiting specificity for diverse tissue ligands (3). However, the precise physiological ligands for each galectin and the physiological concentrations of galectins at the sites of galectinligand interactions are still far from being clearly understood (4).

Among the 19 galectins identified so far, 13 are widely distributed in human tissues, but only a few of them are highly tissuespecific (5). Subsequent to their synthesis as cytosolic proteins, galectins reside in either the cytosol or nucleus and cellular compartments over varying periods of time (6). Because they are devoid of a typical signal peptide, galectins can be exported from cells through a process called non-classical secretion (7) bypassing the Golgi complex (8) and can be found at the cell surface and in the extracellular environment, where they bind to endogenous or exogenous carbohydrate ligands (e.g., glycans from parasites, bacteria, and viruses) $(5,9)$.

In the skin and extracutaneous tissues, galectins are pleiotropic regulators of diverse key biological processes (proliferation, differentiation, apoptosis, signal transduction, cell-cell and cell-extracellular matrix interactions, pre-mRNA splicing, angiogenesis, immune response, tumor migration, and invasion) and are also involved in the pathogenetic mechanisms of various disorders (10-12).

In the last 4 decades there has been increasing interest in galectins among researchers working in reproductive medicine, particularly after recognition of the regulatory effects of these proteins on placentation, maternal-fetal immune response, angiogenesis, and mechanisms underlying the establishment, progression, and maintenance of pregnancy. Although fetal human skin is involved in the maternal-fetal immune response and the production of host defense proteins, and glycoconjugates are known to play significant roles in embryogenesis, knowledge of the expression and significance of galectins in human fetal skin is surprisingly very limited. Thus, we found it of interest to determine the expression of galectins 1, 3, 7, and 9 in the developing epidermis in a series of human embryos at 10 to 23 weeks of gestation and to compare this with the expression observed in adult human epidermis. The main purpose of this comparative study of galectin expression in 
fetal and adult human epidermis can be summarized as follows: a) to define the galectin expression pattern in adult human epidermis because the available data on this subject are either scant or conflicting, thus providing no reliable background for assessment of possible alterations in the diseased human skin, and b) to detect possible differences between already keratinized fetal epidermis at the 23rd week of estimated gestational age (EGA) and adult epidermis with regard to galectin expression pattern.

Among the 13 galectins isolated in human tissues, galectins 1, 3, 7, and 9 were selected for this study for the following reasons: a) they are the most frequently studied galectins in adult human skin, b) the expression of galectins 1 and 3 in fetal human epidermis has been explored in only one study (13), whereas the expression pattern of galectins 7 and 9 in this tissue still remains unknown, and c) all these galectins are involved in important developmental processes during gestation $(14,15)$.

\section{Material and methods}

\section{Skin specimens}

Fifty-eight biopsy skin specimens obtained from the tibiae of morphologically normal human embryos at 10 weeks of gestation $(n=$ $8), 12$ weeks ( $n=11), 14$ weeks $(n=9), 16$ weeks $(n=8), 18$ weeks $(n=10), 20$ weeks $(n=6)$, and 23 weeks $(n=6)$ following legal abortions were included in the study, the protocol for which was approved by the local ethics committee in accordance with the ethical standards laid down in an appropriate version of the 1964 Declaration of Helsinki.

The fetuses were confirmed not to have any obvious skin abnormalities or family history of cutaneous disorders. EGA was determined from maternal histories, fetal measurements (crown, rump, and foot length), and comparative histological appearance of the epidermis (16-19). Biopsy specimens obtained from the buttock skin of 10 healthy adult volunteers of both sexes (age 23-31) were also investigated. All volunteers were informed about the purpose of the study and the possible complications of skin biopsy, and they gave written consent.

All specimens were fixed in buffered formalin (10\%) and embedded in paraffin. One part of the $4 \mu \mathrm{m}$ sections was routinely stained with hematoxylin and eosin and examined histologically, and the other part was processed for galectin immunohistochemistry.

\section{Immunohistochemistry}

The peroxidase-labeled streptavidin-biotin standard technique (20) was applied to $4 \mu \mathrm{m}$ sections of formalin-fixed and paraffinembedded skin specimens using commercially available monoclonal and polyclonal galectin antibodies under the following concentrations: Galectin 1 (Gal 1): 1:100 (mouse) (Novocastra); Galectin 3 (Gal 3): 1:60 (mouse) concentration (NeoMarkers); Galectin 7 (Gal 7): $200 \mu \mathrm{g} / \mathrm{ml}$ (rabbit) 1:200 (Santa Cruz); and Galectin 9 (Gal 9): $200 \mu \mathrm{g} / \mathrm{ml}$ (rabbit) 1:200 (Santa Cruz).

After galectin and the respective secondary biotinylated antibody incubations, in the final step the sections were incubated with chromogen 3,3'-diaminobenzidine $\mathrm{H}_{2} \mathrm{O}_{2}$-containing solution (Sigma, Munich, Germany), their staining intensity was independently graded by two experienced dermatopathologists, and the results of their evaluation were classified on a scale of o to 3 as follows: $0=$ negative stain, $1=$ positive stain of faint to weak in- tensity, 2 = positive stain of moderate intensity, 3 = positive stain of strong intensity.

\section{Statistical analysis}

The results of this study were statistically analyzed with the Jonckheere-Terpstra test $(21,22)$ and the Wilcoxon rank sum test (23) using the software product SAS ${ }^{\circledR}$ (SAS Institute, Cary, North Carolina, USA; version 8.02). The level of significance was fixed at $a=$ $5 \%$. A p-value of 0.05 or less was considered to indicate statistical significance.

\section{Results}

The results of our study are presented in Table 1.

\section{Gal 1}

In all specimens from fetal skin (10th to 23rd week of gestation), all epidermal layers and the basement membrane (BM) revealed no expression of Gal 1. Strong nuclear and cytoplasmic Gal 1 reactivity was observed in spindle mesenchymal cells in the upper dermis (Figs. 1a-1c).

All adult skin epidermal layers and BM exhibited no Gal 1 expression. Spindle mesenchymal cells in the dermis showed strong nuclear and cytoplasmic staining in all specimens investigated. In addition, in the papillary dermis endothelial cells of capillaries revealed a positive reaction (Fig. 1d).

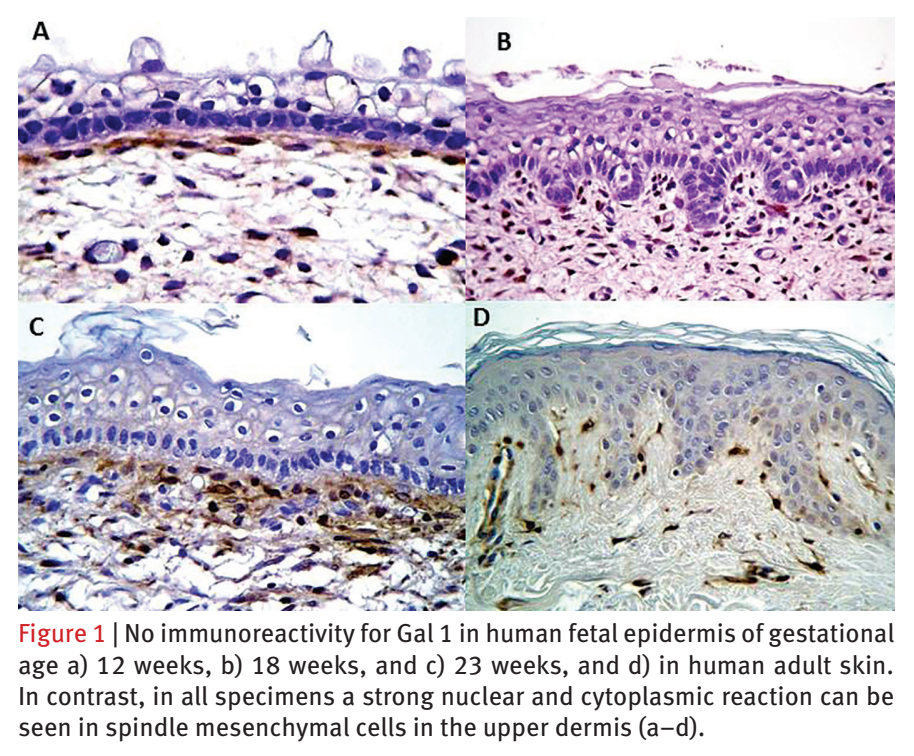

Gal 3

In all specimens from fetal skin (10th to 18th week of gestation), weak to moderate cytoplasmic immunoreactivity for Gal 3 was found in the peridermal cells. No staining was observed in all other epidermal layers and in BM of fetal skin from the 10th to 16th week of gestation (Fig. 2a). At the 18th week only the intermediate layer revealed a positive reaction (weak to moderate; Fig. 2b). At the 2oth week no positive reaction was found in the periderm and BM, whereas the cells in the intermediate and basal layers revealed moderate to strong immunoreactivity for this galectin. At the 23rd week, no positive reaction could be found in all specimens in the horny layer or BM, whereas the cells in the granular, spinous, and basal layers revealed moderate to strong Gal 3 ex- 
pression (Fig. 2C).

Statistically significant differences in the intensity of cytoplasmic immunoreactivity of peridermal cells were found between specimens of the 1oth and 12th week and of the 1oth and 14th week $(p=0.027)$, 10th and 16th week $(p=0.015)$, 10th and 20th week $(p=0.01), 12$ th and 14 th week $(p=0.038)$, 12th and 16th week $(p=0.016), 12$ th and 2oth week $(p<0.001), 14$ th and 2oth week ( $p<$ o.001), 16th and 20th week ( $p=0.020)$, and 18th and 20th week ( $p$ $<$ o.001). Statistically significant differences in the intensity of cytoplasmic immunoreactivity of the intermediate layer were found between specimens of the 18th week and all other gestational ages ( $p \leq 0.0001$ in all cases).

In all adult skin specimens, Gal 3 could not be detected in the

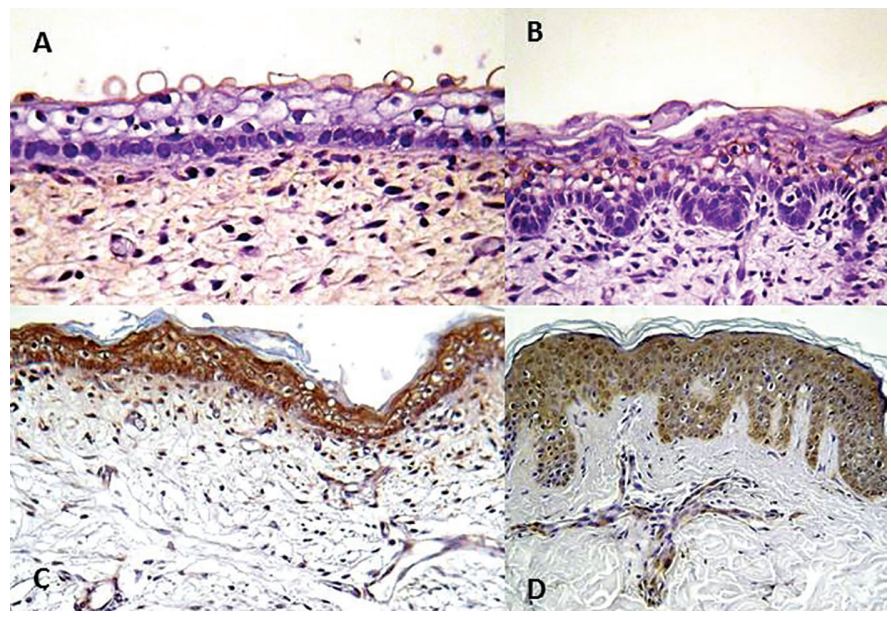

Figure 2 | a) At 14 weeks of estimated gestational age (EGA), weak to moderate cytoplasmic immunoreactivity for Gal 3 was found only in the peridermal cells. b) At the 18th week of EGA, there was additionally a weak to moderate positive reaction in the intermediate layer. c) At the 23 rd week of EGA, no positive reaction could be found in all specimens in the horny layer or in the basement membrane, whereas the cells in the granular, spinous, and basal layers revealed moderate to strong Gal 3 expression. In the adult human epidermis there was moderate to strong cytoplasmic Gal 3 immunoreactivity in the granular layer and in the upper spinous layer, whereas the basal layer revealed weak to strong immunoreactivity. d) In the papillary dermis, there was a moderate positive reaction in the endothelial cells of capillaries and in some mesenchymal cells. horny layer of the epidermis and in BM. In contrast, moderate to strong cytoplasmic expression of Gal 3 was observed in the granular layer and in the upper spinous layer, whereas the basal layer revealed weak to strong immunoreactivity (Fig. 2d). In the papillary dermis, there was a moderate positive reaction in the endothelial cells of capillaries and in some mesenchymal cells.

No statistically significant differences in the immunoreactivity for Gal 3 were found between the adult epidermis and the fetal epidermis at the 23 rd week of gestation.

\section{Gal 7}

In all specimens from fetal skin (10th to 2oth week of gestation) no immunoreactivity for Gal 7 could be detected in the periderm and BM. In contrast, in all fetal specimens cytoplasmic immunostaining of varying intensity was observed in the keratinocytes of the intermediate and basal layers.

At the 1oth week this was limited to suprabasal cells, and its intensity was weak to moderate. At the 12th week there was a weak to strong immunostaining, whereas at the 14 th week a weak to moderate reaction was seen (Fig. 3a). With regard to the basal layer at the 14th and 16th week there was a strong Gal 7 immunostaining of keratinocytes. At the 16th and 18th week, a weak to moderate expression of Gal 7 was found only in the suprabasal cells of the intermediate layer (Fig. 3b). At the 2oth week, a weak reaction was evident in both the intermediate and basal layers of all specimens.

At the 23rd week, a moderate to strong cytoplasmic immunoreactivity for Gal 7 was evident in the granular, spinous, and basal layers of all specimens; the horny layer and the BM revealed no positive reaction (Fig. 3c).

Statistically significant differences in the intensity of cytoplasmic immunoreactivity of the intermediate layer were found between specimens of the 12th and 16th week $(p=0.062)$ and 12th and 2oth week ( $p=0.015)$.

In all adult skin specimens, no immunoreactivity for Gal 7 was observed in the horny layer and BM, whereas the granular, spinous, and basal layers showed a moderate cytoplasmic reac-

Table 1 | Expression pattern of galectins 1, 3, 7, and 9 in human fetal epidermis (gestational age: 10-23 weeks) compared to human adult epidermis.

\begin{tabular}{|c|c|c|c|c|c|c|c|c|c|c|c|c|c|c|c|c|c|c|c|c|c|c|c|c|c|c|c|c|c|c|c|c|c|c|}
\hline \multirow{2}{*}{$\begin{array}{l}\text { GAL } \\
\text { (Abs) }\end{array}$} & \multirow[t]{2}{*}{$\mathbf{E}$} & \multicolumn{4}{|c|}{$\begin{array}{c}10 \text { weeks } \\
(n=8)\end{array}$} & \multicolumn{4}{|c|}{$\begin{array}{c}12 \text { weeks } \\
(n=11)\end{array}$} & \multicolumn{4}{|c|}{$\begin{array}{c}14 \text { weeks } \\
(n=9)\end{array}$} & \multicolumn{4}{|c|}{$\begin{array}{c}16 \text { weeks } \\
(n=8)\end{array}$} & \multicolumn{4}{|c|}{$\begin{array}{c}18 \text { weeks } \\
(n=10)\end{array}$} & \multicolumn{4}{|c|}{$\begin{array}{c}12 \text { weeks } \\
(n=6)\end{array}$} & \multirow[t]{2}{*}{ E } & \multicolumn{4}{|c|}{$\begin{array}{c}23 \text { weeks } \\
(n=6)\end{array}$} & \multicolumn{4}{|c|}{$\begin{array}{c}\text { Adult epidermis } \\
(n=10)\end{array}$} \\
\hline & & 0 & 1 & 2 & 3 & 0 & 1 & 2 & 3 & 0 & 1 & 2 & 3 & 0 & 1 & 2 & 3 & 0 & 1 & 2 & 3 & 0 & 1 & 2 & 3 & & 0 & 1 & 2 & 3 & & 1 & 2 & 3 \\
\hline \multirow{6}{*}{ Gal-1 } & PD & 8 & & 0 & 0 & & 0 & 0 & 0 & 9 & 0 & 0 & 0 & & 0 & 0 & 0 & & & & & & 0 & 0 & 0 & $\mathrm{HL}$ & 6 & 0 & 0 & 0 & 10 & 0 & & 0 \\
\hline & $\begin{array}{l}\text { PD } \\
\text { IL }\end{array}$ & $\begin{array}{l}8 \\
8\end{array}$ & $\begin{array}{l}0 \\
0\end{array}$ & $\begin{array}{l}0 \\
0\end{array}$ & $\begin{array}{l}0 \\
0\end{array}$ & $\begin{array}{l}11 \\
11\end{array}$ & 0 & 0 & 0 & $\begin{array}{l}9 \\
9\end{array}$ & 0 & 0 & 0 & $\begin{array}{l}8 \\
8\end{array}$ & 0 & 0 & 0 & $\begin{array}{l}10 \\
10\end{array}$ & 0 & 0 & 0 & $\begin{array}{l}6 \\
6\end{array}$ & 0 & 0 & 0 & $\mathrm{GL}$ & 6 & 0 & 0 & 0 & 10 & 0 & 0 & \\
\hline & $\mathrm{BL}$ & $\begin{array}{l}0 \\
8\end{array}$ & 0 & 0 & 0 & $\begin{array}{l}11 \\
11\end{array}$ & 0 & 0 & 0 & 9 & 0 & 0 & 0 & $\begin{array}{l}0 \\
8\end{array}$ & 0 & 0 & 0 & & 0 & 0 & 0 & $\begin{array}{l}0 \\
6\end{array}$ & 0 & 0 & 0 & SL & 6 & 0 & 0 & 0 & 10 & 0 & 0 & 0 \\
\hline & BM & 8 & 0 & 0 & 0 & 11 & 0 & 0 & 0 & 9 & 0 & 0 & 0 & 8 & 0 & 0 & 0 & 10 & 0 & 0 & 0 & 6 & 0 & 0 & 0 & BL & 6 & 0 & 0 & 0 & 10 & 0 & 0 & 0 \\
\hline & $\mathrm{SC}$ & 0 & 0 & 0 & 8 & 0 & 0 & 0 & 11 & 0 & 0 & 0 & 9 & 0 & 0 & 0 & 8 & 0 & 0 & 0 & 10 & 0 & 0 & 0 & 6 & BM & 6 & 0 & 0 & 0 & 10 & 0 & 0 & 0 \\
\hline & & & & & & & & & & & & & & & & & & & & & & & & & & SC & 0 & 0 & 0 & 6 & 0 & 0 & 0 & 10 \\
\hline \multirow{5}{*}{ Gal-3 } & PD & 0 & 3 & 5 & 0 & 0 & 5 & 6 & 0 & 0 & 9 & 0 & 0 & 2 & & 0 & 0 & & & 3 & 0 & 6 & 0 & 0 & 0 & $\mathrm{HL}$ & 6 & 0 & 0 & 0 & 10 & 0 & 0 & 0 \\
\hline & IL & 8 & 0 & 0 & 0 & 11 & 0 & 0 & 0 & 9 & 0 & 0 & 0 & 8 & 0 & 0 & 0 & 0 & 3 & 7 & 0 & 0 & 0 & 2 & 4 & $\mathrm{GL}$ & 0 & 0 & 1 & 5 & 0 & 0 & 6 & 4 \\
\hline & $\mathrm{BL}$ & 8 & 0 & 0 & 0 & 11 & 0 & 0 & 0 & 9 & 0 & 0 & 0 & 8 & 0 & 0 & 0 & 10 & & 0 & 0 & 0 & 0 & 3 & 3 & $\mathrm{SL}$ & 0 & 0 & 1 & 5 & 0 & 0 & 7 & 3 \\
\hline & BM & 8 & 0 & 0 & 0 & 11 & 0 & 0 & 0 & 9 & 0 & 0 & 0 & 8 & 0 & 0 & 0 & 10 & 0 & 0 & 0 & 6 & 0 & 0 & 0 & $B L$ & 0 & 0 & 2 & 4 & 0 & 2 & 3 & 5 \\
\hline & & & & & & & & & & & & & & & & & & & & & & & & & & $B M$ & 6 & 0 & 0 & 0 & 10 & 0 & 0 & 0 \\
\hline \multirow{5}{*}{ Gal-7 } & PD & 8 & & 0 & 0 & 11 & & & 0 & & & & 0 & 8 & 0 & 0 & 0 & & & & & & & & & $\mathrm{HL}$ & 6 & 0 & 0 & 0 & 10 & 0 & 0 & 0 \\
\hline & IL & 0 & 5 * & $3 *$ & 0 & 0 & 3 & 6 & 2 & 9 & 6 & 3 & 0 & 0 & 6 * & $2 *$ & 0 & 10 & $5 *$ & 5 * & 0 & 0 & 0 & 0 & 0 & $\mathrm{GL}$ & 0 & 0 & 2 & 4 & 0 & 0 & 10 & 0 \\
\hline & $\mathrm{BL}$ & 0 & 0 & 3 & 5 & 0 & $\begin{array}{l}3 \\
0\end{array}$ & $\begin{array}{l}0 \\
5\end{array}$ & $\begin{array}{l}2 \\
6\end{array}$ & 0 & $\begin{array}{l}0 \\
0\end{array}$ & $\begin{array}{l}3 \\
0\end{array}$ & 9 & 0 & 0 & 0 & 8 & 0 & 10 & 0 & 0 & 0 & $\begin{array}{l}0 \\
6\end{array}$ & 0 & 0 & $\mathrm{SL}$ & 0 & 0 & 4 & 2 & 0 & 0 & 10 & 0 \\
\hline & BM & 8 & 0 & 0 & 0 & 11 & 0 & 0 & 0 & 9 & 0 & 0 & 0 & 8 & 0 & 0 & 0 & & & & 0 & 6 & 0 & 0 & 0 & $\mathrm{BL}$ & 0 & 0 & 3 & 3 & 0 & 0 & 10 & 0 \\
\hline & & & & & & & & & & & & & & & & & & & & & & & & & & $B M$ & 6 & 0 & 0 & 0 & 10 & 0 & 0 & 0 \\
\hline \multirow{5}{*}{ Gal-9 } & PD & 0 & 3 & 5 & 0 & 0 & 3 & 8 & 0 & 0 & 1 & 8 & 0 & 0 & 5 & 3 & 0 & & 4 & 6 & 0 & 0 & 4 & 2 & 0 & $\mathrm{HL}$ & 0 & 1 & 3 & 2 & 10 & 0 & 0 & 0 \\
\hline & IL & 0 & 8 & 0 & 0 & 0 & 9 & 2 & 0 & 0 & $5^{*}$ & $4^{*}$ & 0 & 0 & $4^{*}$ & $4^{*}$ & 0 & 0 & $\begin{array}{l}4 \\
0\end{array}$ & o & $v$ & 0 & 4 & 1 & 0 & $\mathrm{GL}$ & 0 & 1 & 1 & 4 & 0 & 4 & 6 & 0 \\
\hline & $\mathrm{BL}$ & 0 & 8 & 0 & 0 & 0 & 5 & 6 & 0 & 0 & 3 & $\begin{array}{l}4 \\
6\end{array}$ & 0 & 0 & 0 & 3 & 5 & 0 & 0 & 3 & 6 & 0 & ? & 1 & 0 & SL & 0 & 1 & 1 & 4 & 0 & 4 & 6 & 0 \\
\hline & BM & 8 & 0 & 0 & 0 & 11 & 0 & $\begin{array}{l}0 \\
0\end{array}$ & 0 & 9 & $\begin{array}{l}3 \\
0\end{array}$ & $\begin{array}{l}0 \\
0\end{array}$ & 0 & 8 & 0 & 0 & $\begin{array}{l}3 \\
0\end{array}$ & 10 & & 0 & 0 & 6 & $\begin{array}{l}4 \\
0\end{array}$ & $\begin{array}{l}2 \\
0\end{array}$ & 0 & $\mathrm{BL}$ & 0 & 3 & 3 & 0 & 0 & 4 & 6 & 0 \\
\hline & Divi & 0 & 0 & 0 & 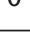 & 11 & 0 & 0 & 0 & 9 & 0 & 0 & 0 & 0 & 0 & 0 & 0 & 10 & & & & 0 & 0 & 0 & 0 & BM & 6 & 0 & 0 & 0 & 10 & 0 & 0 & 0 \\
\hline
\end{tabular}

*mostly in the suprabasal cells.

$\mathrm{GAL}=$ galectin, $\mathrm{Abs}=$ antibodies, $\mathrm{E}=$ epidermis, $\mathrm{PD}=$ periderm, $\mathrm{IL}=$ intermediate layer, $\mathrm{BL}=$ basal layer, $\mathrm{BM}=$ basement $\mathrm{membrane}, \mathrm{HL}=$ horny layer, $\mathrm{GL}=$ granular layer, $\mathrm{SL}=$ spinous layer, $\mathrm{SC}=$ subepidermal cells.

Staining intensity: $0=$ negative, $1=$ faint to weak, $2=$ moderate, $3=$ strong. 
tion (Fig. 3d). Statistically significant differences in the intensity of cytoplasmic immunoreactivity of the basal layer were found between specimens of the 1oth week and adult skin $(p=0.027)$, 10th and 18th week ( $p<0.01)$, 10th and 20th week ( $p=0.01)$, 12th week and adult skin $(p=0.036), 12$ th and 18th week $(p<0.001)$, 12th and 2oth week, 14th week and adult skin, 14th and 18th week ( $p<0.001$ in all cases), 14th and 2oth week ( $p<0.01)$, 14th and 23rd week ( $p=0.0113), 16$ th week and adult skin $(p<0.001), 16$ th and 18th week $(p<0.001), 16$ th and 20th week $(p<0.001)$, 18th week and adult skin $(p<0.001), 18$ th and 23rd week, 20th week and adult skin ( $p<0.001$ in both cases), 20th and 23rd week ( $p=$ o.002), and 23rd week and adult skin ( $p=0.118)$.

Statistically significant differences were also observed between the fetal specimens of the 23 rd week and adult skin in the intensity of cytoplasmic immunostaining of the granular layer $(p=0.031)$ and the spinous layer $(p=0.313)$. Moreover, in all fetal and adult specimens the majority of keratinocytes with cytoplasmic immunoreactivity also revealed a strong nuclear reaction.

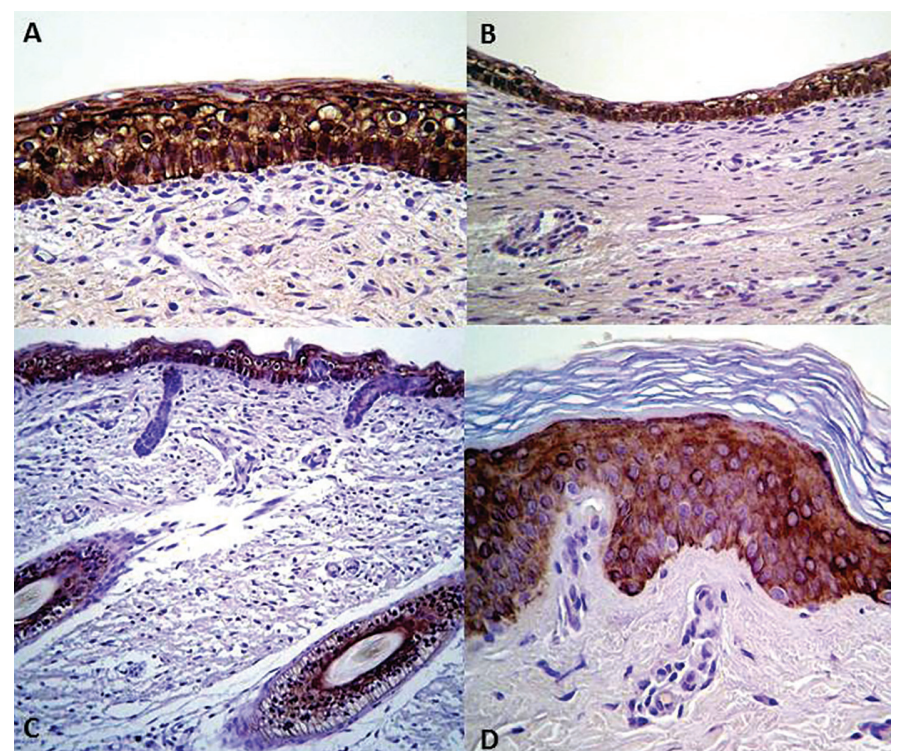

Figure $3 \mid$ a) At the 14 th week of estimated gestational age (EGA), there was a weak to moderate Gal 7 immunoreactivity in the suprabasal cells. b) At the 18th week of EGA, weak to moderate expression of Gal 7 was found only in the suprabasal cells of the intermediate layer. c) At the 23 rd week of EGA, a moderate to strong cytoplasmic immunoreactivity for Gal 7 was evident in the granular, spinous, and basal layers of all specimens, whereas the horny layer and the basement membrane revealed no positive reaction. d) In the adult human epidermis no immunoreactivity for Gal 7 was observed in the horny layer and the basement membrane, whereas the granular, spinous, and basal layers showed a moderate cytoplasmic reaction.

\section{Gal 9}

In all fetal skin specimens (10th to 2oth week of gestation), peridermal cells revealed weak to moderate cytoplasmic immunoreactivity for Gal 9. At the 1oth week of gestation there was a weak and focal cytoplasmic expression of Gal 9 in the basal and intermediate layer, whereas from the 12th to the 16 th week there was a weak to moderate cytoplasmic immunostaining in this layer (Fig. 4a). At the 18th and 2oth week the intensity of cytoplasmic staining in the intermediate layer was weak and mostly weak, respectively (Fig. 4b).

The basal layer revealed a weak cytoplasmic immunoreactivity for Gal 9 at the 1oth week, a weak to moderate immunoreactivity at the 12 th and 14 th week, a moderate to strong immunoreactivity at the 16th week, a weak to strong immunoreactivity at the 18th week, and a focal weak to moderate immunoreactivity at the 2oth week. At the 23rd week, a weak to strong cytoplasmic expression of Gal 9 was detected in the horny, granular, and spinous layers of fetal epidermis, whereas the basal layer revealed a weak to moderate cytoplasmic immunoreactivity (Fig. 4c). In all fetal specimens no immunoreactivity for this galectin was observed in BM.

Statistically significant differences in the intensity of cytoplasmic immunoreactivity of peridermal cells were found between specimens of the 12th and 2oth week $(p=0.216)$ and the 14 th and 2oth week ( $p=0.088$ ). Statistically significant differences in the intensity of cytoplasmic immunoreactivity of the intermediate layer were observed between specimens of the 14 th and 18th week $(p=0.113)$ and the 16th and 18th week ( $p=0.083)$.

The horny layer of adult skin was devoid of any Gal 9 expression, whereas the granular, spinous, and basal layers showed a weak to moderate cytoplasmic immunoreactivity for this galectin. In all adult specimens no staining was found in BM (Fig. 4d).

Statistically significant differences in the intensity of the cytoplasmic immunoreactivity of the basal layer were found between specimens of the 1oth week and adult skin $(p=0.034)$, 10th and 12th week ( $p=0.051)$, 10th and 14 th week $(p=0.021)$, 10th and 16th week $(p<0.001)$, 10th and 18th week $(p=0.001)$, 12th and 16th week $(p=0.003), 12$ th and 18th week $(p=0.006), 14$ th and 16th week $(p=0.008), 14$ th and 18th week $(p=0.017)$, 16th week and adult skin $(p=0.004), 16$ th and 20th week $(p=0.005), 16$ th and 23rd week ( $p=0.008$ in both cases), 18th week and adult skin ( $p=0.011), 18$ th and 20th week $(p=0.011)$, and 18th and 23rd week ( $p=0.022$ in both cases). Moreover, statistically significant differences were found between specimens of the 23rd week and those of adult skin in the intensity of the cytoplasmic staining of the horny layer $(p<0.001)$, and the granular and spinous layers $(p=$ 0.042 in both cases).

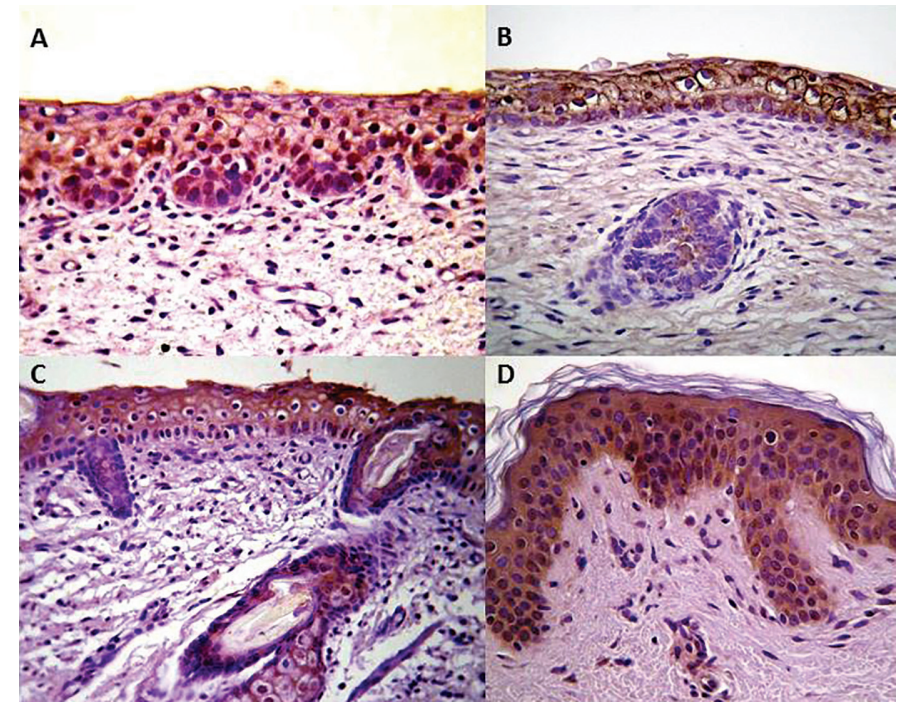

Figure $4 \mid$ a) At the 16 th week of estimated gestational age (EGA), there was a weak to moderate cytoplasmic immunostaining in the periderm and the intermediate layer, whereas the basal layer revealed a moderate to strong reaction. b) At the 18th week of EGA, the intensity of cytoplasmic staining in the intermediate layer was weak, whereas staining of the other layers remained unchanged. c) At the 23rd week of EGA, a weak to strong cytoplasmic expression of Gal 9 was detected in the horny, granular, and spinous layers of fetal epidermis, whereas the basal layer revealed weak to moderate cytoplasmic immunoreactivity. d) In the adult human epidermis, the horny layer and the basement membrane were devoid of any Gal 9 expression, whereas the granular, spinous, and basal layers showed a weak to moderate cytoplasmic immunoreactivity for Gal 9.

\section{Discussion}

Epidermal morphogenesis is a dynamic process involving a com- 
plex network of signaling pathways that interact with each other and contribute to the progressive creation of the shape and structure of epidermis. The study of human epidermal morphogenesis may make it possible to gain better insight into the underlying mechanisms at the molecular and cellular levels and may lead to significant advancement in the prenatal diagnosis of various genetic skin disorders (24).

Epidermal morphogenesis can be divided into the embryonic period (until the 8th week of EGA) and the fetal period (from the 9th week EGA). The latter is subdivided into the epidermal stratification period (9th to $14^{\text {th }}$ week of EGA) and the interfollicular keratinization period ( $14^{\text {th }}$ to 24 th week of EGA). Human epidermis initially consists of a single layer of cuboid basal cells. The periderm is formed during the 4 th week of gestation as a singlecell layer of epithelium situated immediately above the basal layer of the epidermis. Around the 9th week of gestation the intermediate cell layer is formed below the periderm; thus, from the 1oth to the 2oth week of EGA fetal epidermis consists of three distinct epithelial layers: the periderm, intermediate, and basal layers. At the 23rd week of gestation, the periderm is sloughed from the epidermal surface into the amniotic fluid and the fetal epidermis consists of four epidermal layers: the horny, granular, spinous, and basal layers (24).

To our knowledge, only one study has previously explored the expression of galectins during human epidermal morphogenesis. Indeed, in 1997, van den Brule et al. (13) investigated the expression of galectins 1 and 3 in the skin and other tissues and organs in a series of 16 normal human embryos at 6 to 14 weeks of gestation using Western blotting and immunocytochemistry. These authors detected Gal 1 in human fetal skin protein extracts in increasing amounts from 10 weeks to 14 weeks of EGA; however, they were unable to find any Gal 1 immunoreactivity in fetal epidermal cells of 11-week embryos, whereas mesenchymal cells and the extracellular matrix were weakly positive, a finding that explains the positive results of Western blotting. At 14 weeks, basal epidermal cells revealed expression of Gal 1, whereas no immunoreactivity for this galectin was observed in all other epidermal cell layers (25). Based on their findings, these authors suggested that Gal 1 may be implicated in the mechanisms of differentiation of human fetal epidermis.

This study examined the expression of four different galectins (Gal 1, Gal 3, Gal 7, and Gal 9) in the developing epidermis in a series of 58 morphologically normal human embryos 10 to 23 weeks of EGA. Moreover, we compared our findings with those observed in adult human epidermis using biopsy specimens obtained from the buttock skin of 10 healthy volunteers of both sexes (aged 2331).

In contrast to the results of van den Brule et al. (13), our study found that in all specimens, from either fetal skin (10th to 23rd week of EGA) or adult skin, all epidermal layers and BM revealed no Gal 1 expression, whereas strong nuclear and cytoplasmic reactivity was observed in spindle mesenchymal cells in the upper dermis. Our findings with regard to the Gal 1 expression pattern of the human adult epidermis confirm those reported by Lacina et al. (26) and Cada et al. (27), but contradict those of Akimoto et al. (28) and Holikova et al. (29), who described distinct Gal 1 immunoreactivity of keratinocytes of the basal and spinous layers. It is possible that this discrepancy may be at least partially due to differences in the methodological procedures applied. At first glance, the lack of Gal 1 expression in human fetal epidermis observed in our study indicates that this galectin may not be implicated in the mechanisms underlying epidermal morphogenesis. Nevertheless, the possibility that Gal 1 may be capable of indirectly influencing epidermal morphogenesis through interactions of epithelial cells and the underlying mesenchymal cells (which strongly express this galectin) cannot be definitely ruled out.

Van den Brule et al. (13) found that in human embryos during the first trimester of embryogenesis Gal 3 was constantly expressed in all cell layers of the epidermis from 6 to 14 weeks of EGA and also postulated that Gal 3 may participate in the complex process of human epidermal differentiation.

In this study, in all specimens from fetal skin (10th to 18th week of EGA) a strong to moderate immunoreactivity for Gal 3 was found in the peridermal cells. In disagreement with the results of van den Brule et al. (13), we observed a negative reaction in all other epidermal layers and in BM of fetal skin from the 1oth to 16 th week of EGA; however, at the 18th week of EGA a weak to moderate Gal 3 immunoreactivity appeared only in the intermediate layer. At the 2oth week of EGA the periderm (together with BM) revealed a negative reaction, whereas the cells in the intermediate and basal layers revealed a moderate to strong immunoreactivity for this galectin. Finally, in the specimens of the 23rd week of EGA, the pattern of Gal 3 expression was almost identical to that of human adult epidermis (a negative reaction in the horny layer and moderate to strong Gal 3 immunoreactivity in the cells of the granular, spinous, and basal layers). Our findings with regard to the Gal 3 immunoreactivity pattern of human adult epidermis confirm the results previously reported by other research groups (30-32). The distinct qualitative and quantitative changes in Gal 3 immunoreactivity of human fetal epidermis with advancing gestational age are reported here for the first time, most probably reflect corresponding alterations of its glycoconjugate-binding partners, and suggest that this galectin and its ligands may be implicated in the molecular events underlying human epidermal morphogenesis.

Cloning, expression, and chromosome mapping of human Gal 7 was reported for the first time by Madsen et al. (33). Using a Gal 7-specific polyclonal antibody, they observed immunofluorescence staining of all cell layers in fetal epidermis (of visibly advanced but unknown gestational age), which was more intense in the basal layer. However, apart from this observation, no data are available yet with regard to the expression of Gal 7 in human epidermal morphogenesis.

This study reports for the first time the pattern of Gal 7 expression at various stages of human epidermal morphogenesis. In all specimens from fetal skin (10th to 2oth week of EGA), no Gal 7 immunoreactivity was found in the peridermal cells, whereas keratinocytes of the intermediate and basal layer of fetal epidermis revealed a positive reaction, which in most cases was more intense in the basal cells, a finding consistent with the observation by Madsen et al. (33). Finally, in the specimens of the $23 \mathrm{rd}$ week of EGA, the pattern of Gal 7 expression was almost identical to that of the human adult epidermis (horny layer negative and all underlying layers positive). Our findings with regard to the Gal 7 expression pattern of human adult epidermis are in agreement with those reported by Akimoto et al. (28), who suggested that, in view of Gal 7 expression in both the epidermis and in dermal components, this galectin is important for cell-cell contact and/or adhesion in the epidermis and for cell-extracellular matrix interactions in the dermis. Our findings are also in line with the results of a study by Choi et al. (34), who provided evidence suggesting that Gal 7 is a sensitive marker for differentiated and active keratinocytes of human adult epidermis, in which its expression 
reveals a progressive age-dependent decrease.

The importance of Gal 7 as a differentiation marker of epidermal keratinocytes remains in dispute. In keratinocyte cultures, the retinoic acid-induced downregulation of this galectin and the increase of its mRNA in relation to cell density $(35,36)$ have been interpreted in terms of its association with epidermal keratinocyte differentiation. On the other hand, considering Gal 7 expression in and secretion by proliferating basal keratinocytes and differentiated suprabasal keratinocytes (37), this galectin has been regarded by some authors as a marker of stratified epithelia but not as a differentiation marker (38). This view is supported by the fact that our study was unable to detect any evidence indicating a clear-cut relationship between the intensity of Gal 7 immunoreactivity in keratinocytes of either the fetal or adult human epidermis and their differentiation level.

To the best of our knowledge, no data are yet available with regard to the expression of Gal 9 in either the fetal or adult human epidermis. This study reports for the first time the expression pattern of Gal 9 during human epidermal morphogenesis. In all specimens from fetal skin (10th to 2oth week of EGA), the peridermal cells and the cells of the intermediate and basal layers revealed a weak to moderate Gal 9 immunoreactivity, whereas the BM showed a negative reaction. Finally, in all specimens of the 23rd week of EGA, Gal 9 immunoreactivity was observed in all epidermal layers. The only difference from the Gal 9 expression pattern in human adult epidermis was the absence of a positive reaction in the horny layer of adult epidermis.

\section{Conclusions}

The results of this immunohistochemical investigation show that the expression of galectins in human fetal epidermis is character- ized by: 1) a complete lack of Gal 1 in all layers of fetal epidermis at all stages of gestation-a finding that, at first glance, seems to provide no support for a significant role of this galectin in human epidermal morphogenesis. It is possible, however, that Gal 1 may be capable of indirectly influencing epidermal morphogenesis through interactions of epithelial cells with the underlying mesenchymal cells that strongly express this galectin; 2) a constant expression pattern of Gal 7 and Gal 9 at all stages of gestation. The question as to whether, despite this observation, these two galectins may be implicated in the mechanisms of human epidermal morphogenesis remains to be elucidated; 3) distinct qualitative and quantitative changes in Gal 3 immunoreactivity of human fetal epidermis with advancing gestational age, which are reported here for the first time and strongly suggest that this galectin and its ligands may be implicated in the molecular events underlying human epidermal morphogenesis; and 4) an expression pattern of galectins in the already keratinized developing human epidermis at the 23rd week of EGA that is very similar or almost identical to that of the adult human epidermis.

Recent accumulating evidence suggests that galectins 1, 3, 7, and 9 are capable of playing a significant role in the pathogenesis, prognosis, and treatment of diverse skin disorders and also as a potential diagnostic marker $(11,12)$. In view of the reported specific alterations of the galectin 3 pattern in the lesional epidermis of psoriatic patients (39), the prospects for the use of galectins in the prenatal diagnosis of psoriasis and other genodermatoses seem realistic. Thus, it remains to be elucidated in future investigations whether the expression pattern of galectins-particularly that of Gal 3-in the developing human epidermis, as found in this study, reveals alterations in fetuses with inherited cutaneous disorders that may be important for prenatal diagnosis of these disorders.

\section{References}

1. Barondes SH, Cooper DN, Gitt MA, Leffler H. Galectins: structure and function of a large family of animal lectins. J Biol Chem. 1994;269:20807-10.

2. Pasmatzi E, Papadionysiou Ch, Badavanis G, Monastirli A, Tsambaos D. Galectin 3: an extraordinary multifunctional protein in dermatology: current knowledge and perspectives. Anal Bras Dermatol. 2019;94:348-54.

3. Ahmad N, Gabius HJ, Sabesan S, Oscarson S, Brewer SF. Thermodynamic binding studies of bivalent oligosaccharides to galectin-1, galectin-3, and the carbohydrate recognition domain of galectin-3. Glycobiology. 2004;14:817-25.

4. Cummings RD, Liu FT, Gerardo R. Vasta GR. Galectins. In: Varki A, Cummings RD, Esko JD, et al., editors. Essentials of glycobiology. Cold Spring Harbor, NY: Cold Spring Harbor Laboratory Press; 2017.

5. Cooper D. Galectinomics: finding themes in complexity. Biochim Biophys Acta. 2002;1572:209-31.

6. Dings R, Miller M, Griffin R, Mayo K. Galectins as molecular targets for therapeutic intervention. Int J Mol Sci. 2018;19:905.

7. Seelenmeyer C, Wegehingel S, Tews I, Künzler M, Aebi M, Nickel W. Cell surface counter receptors are essential components of the unconventional export machinery of galectin-1. J Cell Biol. 2005;171:373-81.

8. Cummings RD, Liu FT, Vasta GR. Galectins. In: Varki A, Cummings RD, Esko JD, et al., editors. Essentials of glycobiology; 2015. Cold Spring Harbor, NY: Cold Spring Harbor Laboratory Press. p. 469-80.

9. Liu FT, Bevins CL. A sweet target for innate immunity. Nat Med. 2010;16:263-4.

10. Arthur C, Baruffi M, Cummings R, Stowell R. Evolving mechanistic insights into galectin functions. Methods Mol Biol. 2015;1207:1-35.

11. Pasmatzi E, Monastirli A, Badavanis G, Papadionysiou C, Tsambaos D. Galec tins 7 and 9 in dermatology: current knowledge and future perspectives. Hosp Chron. 2018;13:19-26.

12. Pasmatzi E, Papadionysiou C, Badavanis G, Monastirli A, Tsambaos D. Galectin 1 in dermatology: current knowledge and perspectives. Acta Dermatovenereol Alp Pannonica Adriat. 2019;28:27-31.
13. van de Brule F, Fernandez P, Buicu C, Liu F, Jackers P, Lambotte R, et al. Differential expression of galectin-1 and galectin-3 during first trimester human embryogenesis. Dev Dyn. 1997;209:399-405.

14. Menkhorst E, Gamage T, Cuman C, Kaitu'u-Lino T, Tong S, Dimitriadis E. Galectin-7 acts as an adhesion molecule during implantation and increased expression is associated with miscarriage. Placenta. 2014;35:195-201.

15. Conrad M, Freitag N, Diessler M, Hernandez R, Barrientos G, Rose M, et al. Differential spatiotemporal patterns of galectin expression are a hallmark of endotheliochorial placentation. Am J Reprod Immunol. 2016;75:317-25.

16. Trolle D. Age of fetus determined from its measures. Acta Obstet Gynecol Scand. 1948;27:327-37.

17. Shepard T. Normal and abnormal growth patterns. Growth and development of the human embryo and fetus. In: Gardner LI, editor. Endocrine and genetic diseases of childhood and adolescence. Philadelphia: WB Saunders; 1975. p. 1-8.

18. Holbrook KA. Human epidermal embryogenesis. Int J Dermatol. 1979;18:32956.

19. Mercer BM, Sklar S, Shariatmadar A, Gillieson MS, D’Alton ME. Fetal foot length as a predictor of gestational age. J Obstet Gynecol. 1987;156:350-5.

20. Monastirli A, Vourekas A, Badavanis G, Pasmatzi E, Sagriotis A, Drainas D, et al. Hsp27 expression coincides with epidermal stratification during human epidermal morphogenesis. Acta Derm Venereol. 2005;85:389-93.

21. Hollander M, Wolfe DA. Nonparametric statistical methods. New York: John Wiley \& Sons; 1979.

22. Pirie W. Jonckheere tests for ordered alternatives. In: Kotz S, Johnson NL, editors. Encyclopedia of statistical sciences, vol 4. New York: John Wiley \& Sons; 1983. p. 315-8.

23. Sprent P. Applied nonparametric statistical methods. 2nd ed. London: Chapman \& Hall; 1993.

24. Badavanis G, Tsambaos D. Morphogenesis of the periderm. Dermatopathol. 2002;3:1-5. 
25. Pasmatzi E, Badavanis G, Monastirli A, Georgiou S, Sagriotis A, Sakkis T, et al. Qualitative and quantitative alterations of cell surface carbohydrate residues during epidermal morphogenesis. Anat Embryol (Berl). 2005;209:207-15.

26. Lacina L, Plzakova Z, Smetana K, Stork J, Kaltner H, Andre S. Glycophenotype of psoriatic skin. Folia Biol (Praha). 2006;52:10-5.

27. Cada Z, Chovanec M, Smetana K, Betka J, Lacina L, Plzák J, et al. Galectin-7: will the lectin's activity establish clinical correlations in head and neck squamous cell and basal cell carcinomas? Histol Histopathol. 2009;24:41-8.

28. Akimoto Y, Hirabayashi J, Kasai K, Hirano H. Expression of the endogenous 14 $\mathrm{kDa}$ beta-galactoside-binding lectin galectin in normal human skin. Cell Tissue Res. 1995;280:1-10.

29. Holikova Z, Hrdlickova-Cela E, Plzak J, Smetana K Jr, Betka J, Dvorankova B, et al. Defining the glycophenotype of squamous epithelia using plant and mammalian lectins. Differentiation-dependent expression of alpha2,6- and alpha2,3linked $\mathrm{N}$-acetylneuraminic acid in squamous epithelia and carcinomas and its differential effect on binding of the endogenous lectins galectins-1 and -3 . APMIS. 2002;110:845-56.

30. Konstantinov KN, Shames B, Izuno G, Liu FT. Expression of epsilon BP, a betagalactoside-binding soluble lectin, in normal and neoplastic epidermis. Exp Dermatol. 1994:3:9-16.

31. Nangia-Makker P, Honjo Y, Sarvis R, Akahani S, Hogan V, Pienta KJ, et al. Galectin-3 induces endothelial cell morphogenesis and angiogenesis. Am J Pathol. 2000;156:899-909.
32. Larsen L, Chen HH, Saegusa J, Liu FT. Galectin-3 and the skin. J Derm Sci. 2011; 64:85-91.

33. Madsen P, Rasmussen HH, Flint T, Gromov P, Kruse TA, Honore B, et al. Cloning, expression, and chromosome mapping of human galectin-7. J Biol Chem. 1995; 270:5823-9.

34. Choi JW, Nam KM, Choi HR, Lee DH, Huh CH, Park KC. Decreased galectin-3 and -7 expressions in old-aged skin and their differential expression in skin equivalents. Ann Dermatol. 2018;30:375-8.

35. Magnaldo T, Bernerd F, Darmon M. Galectin-7, a human 14-kDa S-lectin, specifically expressed in keratinocytes and sensitive to retinoic acid. Dev Biol. 1995; 168:259-71.

36. Polyak K, Xia Y, Zweier, J, Kinzler K, Vogelstein B. A model for p53-induced apoptosis. Nature (London). 1997;389:300-5.

37. Magnaldo T, Fowlis D, Darmon, M. Galectin-7, a marker of all types of stratified epithelia. Differentiation. 1998;63:159-68.

38. Advedissian T, Proux-Gillardeaux V, Nkosi R, Peyret G, Nguyen T, Poirier F, et al. E-cadherin dynamics is regulated by galectin-7 at epithelial cell surface. Sci Rep. 2017;7:17086.

39. Shi Z, Tan G, Tao C, Han Y, Meng Z, Man X, et al. Decrease of galectin 3 in keratinocytes: a potential diagnostic marker and a critical contributor to the pathogenesis of psoriasis. J Autoimmun. 2018;89:30-40. 